\title{
Linguistic Intuitionistic Fuzzy Sets and Application in MAGDM
}

\author{
Huimin Zhang \\ School of Management, Henan University of Technology, Zhengzhou 450001, China \\ Correspondence should be addressed to Huimin Zhang; zhm76@126.com \\ Received 19 January 2014; Revised 5 April 2014; Accepted 8 April 2014; Published 29 April 2014 \\ Academic Editor: Reinaldo Martinez Palhares \\ Copyright (C) 2014 Huimin Zhang. This is an open access article distributed under the Creative Commons Attribution License, \\ which permits unrestricted use, distribution, and reproduction in any medium, provided the original work is properly cited. \\ To better deal with imprecise and uncertain information in decision making, the definition of linguistic intuitionistic fuzzy \\ sets (LIFSs) is introduced, which is characterized by a linguistic membership degree and a linguistic nonmembership degree, \\ respectively. To compare any two linguistic intuitionistic fuzzy values (LIFVs), the score function and accuracy function are \\ defined. Then, based on $t$-norm and $t$-conorm, several aggregation operators are proposed to aggregate linguistic intuitionistic \\ fuzzy information, which avoid the limitations in exiting linguistic operation. In addition, the desired properties of these linguistic \\ intuitionistic fuzzy aggregation operators are discussed. Finally, a numerical example is provided to illustrate the efficiency of the \\ proposed method in multiple attribute group decision making (MAGDM).
}

\section{Introduction}

Intuitionistic fuzzy set (IFS) [1], which is characterized by a degree of membership and a degree of nonmembership, is a very powerful tool to process vague information. After the pioneering study of Atanassov [1], the IFS has captured much attention from researchers in various fields and many achievements have been made, such as entropy measure of IFS [2-7], distance, or similarity measure between IFSs [813] and aggregation operators of IFS [14-21]. In addition, related to IFS, some authors proposed several other tools to handle vague and imprecise information whereby two or more sources of vagueness appear simultaneously [22]. Atanassov and Gargov [23] introduced the notion of intervalvalued intuitionistic fuzzy set (IVIFS), which is characterized by a membership function and a nonmembership function with interval values. Torra [24] and Torra and Narukawa [25] gave a definition of hesitant fuzzy set (HFS), which can better deal with the situations where several values are possible to determine the membership of an element. Zhu et al. [26] defined dual hesitant fuzzy set in terms of two functions that return two sets of membership values and nonmembership values, respectively, for each element in the domain.

Although, the foregoing fuzzy tools are suitable for dealing with problems that are defined as quantitative situations [22], uncertainty is often because of the vagueness of meanings that is used by experts in problems whose nature is rather qualitative. For example, for reason of the increasing complexity of the decision making environment, time pressure, and the lack of data or knowledge about the problem domain, in the process of decision making under intuitionistic fuzzy environment, a decision maker may have difficulty in expressing the degree of membership and nonmembership as exact values, whereas he or she may think the use of linguistic values is more straightforward and suitable to express the degree of membership and nonmembership. Similar to IFS, linguistic intuitionistic fuzzy set (LIFS) is characterized by a linguistic membership degree and a linguistic nonmembership degree, respectively. By using the LIFS, decision makers are able to consider a linguistic hesitancy degree in the belongingness of an element to a set, where they cannot easily express their subjective judgment with a single linguistic term.

The outline of the paper is organized as follows. The following section presents a brief introduction to the basic knowledge that will be used in the definition of LIFS. Section 3 gives the concept of LIFS and constructs the score function and accuracy function for LIFS. Section 4 develops several aggregation operators for LIFS. Section 5 proposes a MAGDM method with linguistic intuitionistic fuzzy information. In Section 6, an application of the new approach is presented. Finally, conclusions are provided in Section 7. 


\section{Preliminaries}

In the following, some basic concepts and knowledge related to IFS and linguistic approach are briefly described.

Definition 1 (see [1]). Let $X$ be a universal set. An IFS $A$ in $X$ is given as

$$
A=\left\{\left(x, \mu_{A}(x), v_{A}(x)\right) \mid x \in X\right\},
$$

where the functions $\mu_{A}(x): X \rightarrow[0,1], v_{A}(x): X \rightarrow[0,1]$ stand for the degree of membership and nonmembership of the element $x$ to $A$, respectively. Any $x \in X$ meets the condition $0 \leq \mu_{A}(x)+v_{A}(x) \leq 1$.

$\pi_{A}(x)$ is called intuitionistic index or degree of indeterminacy of $x$ to $A: \pi_{A}(x)=1-\mu_{A}(x)-v_{A}(x)$. Obviously, if $\pi_{A}(x)=0$, IFS $A$ is reduced to a fuzzy set.

Some basic definitions and operations on IFS are presented as follows.

Definition 2 (see $[14,15]$ ). If $A$ and $B$ are two IFSs of the set $X$, then

(1) $A=B$ if and only if $\forall x \in X, \mu_{A}(x)=\mu_{B}(x)$, and $v_{A}(x)=v_{B}(x)$

(2) $A^{c}=\left\{\left(x, v_{A}(x), \mu_{A}(x)\right) \mid x \in X\right\}$, where $A^{c}$ is the complement of $A$;

(3) $A \wedge B=\left(\min \left(\mu_{A}(x), \mu_{B}(x)\right), \max \left(v_{A}(x), v_{B}(x)\right)\right)$;

(4) $A \vee B=\left(\max \left(\mu_{A}(x), \mu_{B}(x)\right), \min \left(v_{A}(x), v_{B}(x)\right)\right)$;

(5) $A \oplus B=\left(\mu_{A}(x)+\mu_{B}(x)-\mu_{A}(x) \mu_{B}(x), v_{A}(x) v_{B}(x)\right)$;

(6) $A \otimes B=\left(\mu_{A}(x) \mu_{B}(x), v_{A}(x)+v_{B}(x)-v_{A}(x) v_{B}(x)\right)$;

(7) $\lambda A=\left(1-\left(1-\mu_{A}(x)\right)^{\lambda}, v_{A}(x)^{\lambda}\right), \lambda>0$;

(8) $A^{\lambda}=\left(\mu_{A}(x)^{\lambda}, 1-\left(1-v_{A}(x)\right)^{\lambda}\right), \lambda>0$.

In real world, many decision making problems present qualitative aspects that are complex to assess by means of numerical values. In such cases, it may be more suitable to consider them as linguistic variables.

Let $S=\left\{s_{i} \mid i=0,1, \ldots, t\right\}$ be a finite linguistic term set with odd cardinality, where $s_{i}$ represents a possible linguistic term for a linguistic variable. For example, a set of seven terms $S$ can be expressed as follows:

$$
\begin{gathered}
S=\left\{s_{0}=\mathrm{N}(\text { none }), s_{1}=\mathrm{VL}(\text { very low }), s_{2}=\mathrm{L}(\text { low }),\right. \\
s_{3}=\mathrm{M}(\text { medium }), s_{4}=\mathrm{H}(\text { high }), \\
\left.s_{5}=\mathrm{VH}(\text { very high }), s_{6}=\mathrm{P}(\text { perfect })\right\} .
\end{gathered}
$$

It is required that the linguistic term set should satisfy the following characteristics [27-30].

(1) The set is ordered: $s_{i}>s_{j}$, if and only if $i>j$.

(2) There is a negation operator: $\operatorname{Neg}\left(s_{i}\right)=s_{j}$ such that $j=t-i$.

(3) Max operator: $\max \left(s_{i}, s_{j}\right)=s_{i}$, if and only if $i \geq j$.

(4) Min operator: $\min \left(s_{i}, s_{j}\right)=s_{i}$, if and only if $i \leq j$.
To preserve all the given information, $\mathrm{Xu}$ [31] extended the discrete term set $S$ to a continuous linguistic term set $\bar{S}=$ $\left\{s_{\alpha} \mid s_{0}<s_{\alpha} \leq s_{t}, \alpha \in[0, t]\right\}$, where, if $s_{\alpha} \in S$, then $s_{\alpha}$ is called the original linguistic term. Otherwise, $s_{\alpha}$ is called the virtual linguistic term.

Definition 3 (see $[31,32]$ ). Consider any two linguistic terms $s_{\alpha}, s_{\beta} \in \bar{S}$, and $\mu, \mu_{1}, \mu_{2} \in[0,1]$, the add and multiply operations of linguistic variable are defined as follows:

$$
\begin{gathered}
s_{\alpha} \oplus s_{\beta}=s_{\beta} \oplus s_{\alpha}=s_{\alpha+\beta}, \\
s_{\alpha} \otimes s_{\beta}=s_{\beta} \otimes s_{\alpha}=s_{\alpha \beta} .
\end{gathered}
$$

$t$-norm and $t$-conorm have been widely used to construct operations for fuzzy sets and IFSs.

Definition 4 (see $[33,34])$. A $t$-norm is a mapping $T:[0,1] \times$ $[0,1] \rightarrow[0,1]$ satisfying, for all $x, y, z \in[0,1]$,

(1) $T(x, 1)=x$;

(2) $T(x, y)=T(y, x)$;

(3) $T(x, T(y, z))=T(T(x, y), z)$;

(4) $T(x, y) \leq T(x, z)$ whenever $y \leq z$.

The four basic $t$-norms $T_{M}, T_{P}, T_{L}$, and $T_{D}$ are given as follows:

$$
\begin{aligned}
& T_{M}(x, y)=\min (x, y),(\text { lattice operation); } \\
& T_{P}(x, y)=x \cdot y,(\text { algebraic operation); } \\
& T_{L}(x, y)=\max (x+y-1,0),(\text { Lukasiewicz operation); } \\
& T_{D}(x, y)=\left\{\begin{array}{ll}
0 & \text { if }(x, y) \in[0,1)^{2} \\
\min (x, y) & \text { otherwise. }
\end{array}\right. \text { (drastic operation). }
\end{aligned}
$$

Definition 5 (see $[33,34])$. A $t$-conorm is a mapping $S$ : $[0,1] \times[0,1] \rightarrow[0,1]$ satisfying, for all $x, y, z \in[0,1]$,
(1) $S(x, 0)=x$;
(2) $S(x, y)=S(y, x)$;
(3) $S(x, S(y, z))=S(S(x, y), z)$;
(4) $S(x, y) \leq S(x, z)$ whenever $y \leq z$. follows:

$$
\begin{aligned}
& S_{M}(x, y)=\max (x, y),(\text { lattice operation); } \\
& S_{P}(x, y)=x+y-x \cdot y,(\text { algebraic operation); } \\
& S_{L}(x, y)=\min (x+y, 1),(\text { Lukasiewicz operation); } \\
& S_{D}(x, y)=\left\{\begin{array}{ll}
\max (x, y) & \text { if }(x, y) \in(0,1]^{2} \\
1 & \text { otherwise. }
\end{array}\right. \text { (drastic operation). }
\end{aligned}
$$

\section{Linguistic Intuitionistic Fuzzy Set}

The concept of linguistic intuitionistic fuzzy set (LIFS) is given as follows. 
Definition 6. Let $X$ be a finite universal set and $\bar{S}=\left\{s_{\alpha} \mid s_{0}<\right.$ $\left.s_{\alpha} \leq s_{t}, \alpha \in[0, t]\right\}$ a continuous linguistic term set. A LIFS $A$ in $X$ is given as

$$
A=\left\{\left(x, s_{\theta}(x), s_{\sigma}(x)\right) \mid x \in X\right\},
$$

where $s_{\theta}(x), s_{\sigma}(x) \in \bar{S}$ stand for the linguistic membership degree and linguistic nonmembership of the element $x$ to $A$, respectively.

For any $x \in X$, the condition $0 \leq \theta+\sigma \leq t$ is always satisfied. $\pi(x)$ is called linguistic indeterminacy degree of $x$ to $A: \pi(x)=s_{t-\theta-\sigma}$. Obviously, if $\theta+\sigma=t$, then LIFS $A$ has the minimum linguistic indeterminacy degree, that is, $\pi(x)=s_{0}$, which means the membership degree of $x$ to $A$ can be precisely expressed with a single linguistic term and LIFS $A$ is reduced to a linguistic variable. Oppositely, if $\theta=\sigma=0$, then LIFS $A(x)$ has the maximum linguistic indeterminacy degree; that is, $\pi(x)=s_{t}$. Similar to IFS, the LIFS $A(x)$ can be transformed into an interval linguistic variable $\left[s_{\theta}(x), s_{t-\sigma}(x)\right]$, which indicates that the minimum and maximum linguistic membership degrees of the elements $x$ to $A$ are $s_{\theta}$ and $s_{t-\sigma}$, respectively.

For notational simplicity, we suppose both LIFS $A$ and $B$ contain only one element, which stand for linguistic intuitionistic fuzzy values (LIFVs), that is, the pairs $A=$ $\left(s_{\theta}, s_{\sigma}\right)$ and $B=\left(s_{\mu}, s_{\gamma}\right)$.

To compare any two LIFVs, the score function and accuracy function are defined as follows.

Definition 7. Let $A=\left(s_{\theta}, s_{\sigma}\right)$ and $B=\left(s_{\mu}, s_{\nu}\right)$ be two LIFVs, with $s_{\theta}, s_{\sigma}, s_{\mu}, s_{\nu} \in \bar{S}=\left\{s_{\alpha} \mid s_{0}<s_{\alpha} \leq s_{t}, \alpha \in[0, t]\right\}$. The score function of $A$ is defined as

$$
S(A)=s_{(t+\theta-\sigma) / 2},
$$

and the accuracy function is defined as

$$
H(A)=s_{\theta+\sigma} .
$$

Thus, $A$ and $B$ can be ranked by the following procedure:

(1) if $S(A)>S(B)$, then $A>B$;

(2) if $S(A)=S(B)$ and
(a) $H(A)=H(B)$, then $A=B$;
(b) $H(A)>H(B)$, then $A>B$;
(c) $H(A)<H(B)$, then $A<B$.

It is easy to see that $0 \leq(t+\theta-\sigma) / 2 \leq t$ and $0 \leq \theta+\sigma \leq t$, which means $s_{(t+\theta-\sigma) / 2}, s_{\theta+\sigma} \in \bar{S}$.

Example 8. Let $A=\left(s_{1}, s_{3}\right), B=\left(s_{0}, s_{4}\right)$, and $C=\left(s_{1}, s_{5}\right)$ be LIFVs, which are derived from $\bar{S}=\left\{s_{\alpha} \mid s_{0}<s_{\alpha} \leq s_{6}, \alpha \in\right.$ $[0,6]\}$.

Applying formulas (5) and (6), we have

$$
\begin{gathered}
S(A)=s_{2}>S(B)=S(C)=s_{1}, \\
H(B)=s_{4}<H(C)=s_{6} .
\end{gathered}
$$

Thus, we obtain $A>C>B$.

\section{Aggregation Operators for Linguistic Intuitionistic Fuzzy Sets}

Since the definition of LIFS is given, it is necessary to introduce the operations and computations between them.

Definition 9. Let $A=\left(s_{\theta}, s_{\sigma}\right)$ and $B=\left(s_{\mu}, s_{\nu}\right)$ be two LIFVs; then

(1) $A=B$ if and only if $s_{\theta}=s_{\mu}$ and $s_{\sigma}=s_{\gamma}$;

(2) $A^{c}=\left(s_{\sigma}, s_{\theta}\right)$, where $A^{c}$ is the complement of $A$;

(3) the intersection of $A$ and $B: A \wedge B=$ $\left(\min \left(s_{\theta}, s_{\mu}\right), \max \left(s_{\sigma}, s_{\nu}\right)\right)$;

(4) the union of $A$ and $B: A \vee B=$ $\left(\max \left(s_{\theta}, s_{\mu}\right), \min \left(s_{\sigma}, s_{\nu}\right)\right)$.

Motivated by $t$-norm and $t$-conorm, we propose the following operation laws for linguistic variables.

Definition 10. Considering any two linguistic terms $s_{\alpha}, s_{\beta} \in$ $\bar{S}=\left\{s_{\gamma} \mid s_{0}<s_{\gamma} \leq s_{t}, \gamma \in[0, t]\right\}$, the add and multiply operations of linguistic variable are defined as follows:

$$
\begin{aligned}
& s_{\alpha} \oplus s_{\beta}=s_{\beta} \oplus s_{\alpha}=s_{t S(\alpha / t, \beta / t)}, \\
& s_{\alpha} \otimes s_{\beta}=s_{\beta} \otimes s_{\alpha}=s_{t T(\alpha / t, \beta / t)},
\end{aligned}
$$

where $S(\alpha / t, \beta / t)$ and $T(\alpha / t, \beta / t)$ are $t$-conorm and $t$-norm, respectively.

Since $S(\alpha / t, \beta / t), T(\alpha / t, \beta / t) \in[0,1]$, we have $t S(\alpha / t$, $\beta / t), t T(\alpha / t, \beta / t) \in[0, t]$, which indicate the operation results match the original linguistic term set $\bar{S}$; that is, $s_{t S(\alpha / t, \beta / t)}, s_{t T(\alpha / t, \beta / t)} \in \bar{S}$. In addition, it is worth noting that, because of the monotonicity of $t$-conorm and $t$-norm, the values of function $t S(\alpha / t, \beta / t)$ and $t T(\alpha / t, \beta / t)$ are monotonically increasing with the increasing of $\alpha$ and $\beta$, which means the operation results obtained by (8) and (9) are in accord with our intuition.

If we take the well-known $S_{P}(\alpha / t, \beta / t)$ and $T_{P}(\alpha / t, \beta / t)$ into (8) and (9), respectively, then they can be rewritten as follows:

$$
\begin{gathered}
s_{\alpha} \oplus s_{\beta}=s_{\beta} \oplus s_{\alpha}=s_{t\left(\alpha / t+\beta / t-\alpha \beta / t^{2}\right)}=s_{\alpha+\beta-\alpha \beta / t}, \\
s_{\alpha} \otimes s_{\beta}=s_{\beta} \otimes s_{\alpha}=s_{t\left(\alpha \beta / t^{2}\right)}=s_{\alpha \beta / t} .
\end{gathered}
$$

Example 11. Let $\bar{S}=\left\{s_{\alpha} \mid s_{0}<s_{\alpha} \leq s_{6}, \alpha \in[0,6]\right\}$. Applying (10), we have $s_{3} \oplus s_{4}=s_{3+4-3 \times 4 / 6}=s_{5}, s_{4} \oplus s_{5}=s_{4+5-4 \times 5 / 6}=$ $s_{5.667}, s_{3} \otimes s_{4}=s_{3 \times 4 / 6}=s_{2}$, and $s_{4} \otimes s_{5}=s_{4 \times 5 / 6}=s_{3.333}$.

Thus, we obtain $s_{3} \oplus s_{4}<s_{4} \oplus s_{5}$ and $s_{3} \otimes s_{4}<s_{4} \otimes s_{5}$. Such results seem to be intuitive and can be easily accepted.

Alternatively, if we take the operation laws of Definition 3, we have $s_{3} \oplus s_{4}=s_{7} \notin \bar{S}, s_{4} \otimes s_{5}=s_{20} \notin \bar{S}$, where the subscripts of $s_{7}$ and $s_{20}$ are bigger than the cardinality of linguistic term set $S$. In addition, if we extend the discrete term set $S$ to a continuous term set $\bar{S}^{\prime}=\left\{s_{\alpha} \mid \alpha \epsilon\right.$ $[0, q]\}[35]$, where $q(q>t)$ is a sufficiently large positive 
integer, there is an unavoidable question on how to define the semantics for $s_{7}$ and $s_{20}$. Obviously, $s_{7}$ or $s_{20}$ has different semantics in different linguistic term set $S$ with different cardinalities. As a result, it is unrealistic to assign semantics to a given linguistic value $s_{\alpha}$ derived from linguistic term set with variable cardinality. If we follow the method of $s_{\alpha} \oplus s_{\beta}=$ $\min \left\{s_{\alpha+\beta}, s_{t}\right\}$ and $s_{\alpha} \otimes s_{\beta}=\min \left\{s_{\alpha \beta}, s_{t}\right\}$ [36] for $s_{\alpha}, s_{\beta} \in \bar{S}=$ $\left\{s_{\gamma} \mid s_{0}<s_{\gamma} \leq s_{t}, \gamma \in[0, t]\right\}$, then we have $s_{3} \oplus s_{4}=$ $s_{4} \oplus s_{5}=s_{6}$ and $s_{3} \otimes s_{4}=s_{4} \otimes s_{5}=s_{6}$. Such results seem to be counter-intuitive and may not be easily accepted. Applying (10), we can overcome the limitations that the subscripts of the linguistic variable are bigger than the cardinality of the corresponding linguistic term set $S$ and obtain results agreed with our intuition.

Based on (10), we can get the following operation laws for LIFVs.

Definition 12. Let $A=\left(s_{\theta}, s_{\sigma}\right)$ and $B=\left(s_{\mu}, s_{\nu}\right)$ be two LIFVs, where $s_{\theta}, s_{\sigma}, s_{\mu}, s_{\nu} \in \bar{S}=\left\{s_{\alpha} \mid s_{0}<s_{\alpha} \leq s_{t}, \alpha \in[0, t]\right\}$ with $\lambda>0$; then

$$
\begin{gathered}
A \oplus B=\left(s_{t\left(\theta / t+\mu / t-\theta \mu / t^{2}\right)}, s_{t\left(\sigma \nu / t^{2}\right)}\right)=\left(s_{\theta+\mu-\theta \mu / t}, s_{\sigma \nu / t}\right), \\
A \otimes B=\left(s_{t\left(\theta \mu / t^{2}\right)}, s_{t\left(\sigma / t+v / t-\sigma v / t^{2}\right)}\right)=\left(s_{\theta \mu / t}, s_{\sigma+v-\sigma \nu / t}\right), \\
\lambda A=\left(s_{t\left(1-(1-\theta / t)^{\lambda}\right)}, s_{t(\sigma / t)^{\lambda}}\right), \\
A^{\lambda}=\left(s_{t(\theta / t)^{\lambda}}, s_{t\left(1-(1-\sigma / t)^{\lambda}\right)}\right) .
\end{gathered}
$$

Some special cases of $\lambda A$ and $A^{\lambda}$ are obtained as follows.

If $A=\left(s_{\theta}, s_{\sigma}\right)=\left(s_{t}, s_{0}\right)$, then

$\lambda A=\left(s_{t\left(1-(1-\theta / t)^{\lambda}\right)}, s_{t(\sigma / t)^{\lambda}}\right)=\left(s_{t\left(1-(1-t / t)^{\lambda}\right)}, s_{t(0 / t)^{\lambda}}\right)=\left(s_{t}, s_{0}\right)$,

$A^{\lambda}=\left(s_{t(\theta / t)^{\lambda}}, s_{t\left(1-(1-\sigma / t)^{\lambda}\right)}\right)=\left(s_{t(t / t)^{\lambda}}, s_{t\left(1-(1-0 / t)^{\lambda}\right)}\right)=\left(s_{t}, s_{0}\right)$.

If $A=\left(s_{\theta}, s_{\sigma}\right)=\left(s_{0}, s_{t}\right)$, then

$\lambda A=\left(s_{t\left(1-(1-\theta / t)^{\lambda}\right)}, s_{t(\sigma / t)^{\lambda}}\right)=\left(s_{t\left(1-(1-0 / t)^{\lambda}\right)}, s_{t(t / t)^{\lambda}}\right)=\left(s_{0}, s_{t}\right)$,

$A^{\lambda}=\left(s_{t(\theta / t)^{\lambda}}, s_{t\left(1-(1-\sigma / t)^{\lambda}\right)}\right)=\left(s_{t(0 / t)^{\lambda}}, s_{t\left(1-(1-t / t)^{\lambda}\right)}\right)=\left(s_{0}, s_{t}\right)$.

If $A=\left(s_{\theta}, s_{\sigma}\right)=\left(s_{0}, s_{0}\right)$, then

$\lambda A=\left(s_{t\left(1-(1-\theta / t)^{\lambda}\right)}, s_{t(\sigma / t)^{\lambda}}\right)=\left(s_{t\left(1-(1-0 / t)^{\lambda}\right)}, s_{t(0 / t)^{\lambda}}\right)=\left(s_{0}, s_{0}\right)$,

$A^{\lambda}=\left(s_{t(\theta / t)^{\lambda}}, s_{t\left(1-(1-\sigma / t)^{\lambda}\right)}\right)=\left(s_{t(0 / t)^{\lambda}}, s_{t\left(1-(1-0 / t)^{\lambda}\right)}\right)=\left(s_{0}, s_{0}\right)$.

If $\lambda \rightarrow 0$, then

$$
\begin{aligned}
& \lambda A=\left(s_{t\left(1-(1-\theta / t)^{\lambda}\right)}, s_{t(\sigma / t)^{\lambda}}\right)=\left(s_{0}, s_{t}\right), \\
& A^{\lambda}=\left(s_{t(\theta / t)^{\lambda}}, s_{t\left(1-(1-\sigma / t)^{\lambda}\right)}\right)=\left(s_{t}, s_{0}\right) .
\end{aligned}
$$

If $\lambda \rightarrow+\infty$, then

$$
\begin{aligned}
& \lambda A=\left(s_{t\left(1-(1-\theta / t)^{\lambda}\right)}, s_{t(\sigma / t)^{\lambda}}\right)=\left(s_{t}, s_{0}\right), \\
& A^{\lambda}=\left(s_{t(\theta / t)^{\lambda}}, s_{t\left(1-(1-\sigma / t)^{\lambda}\right)}\right)=\left(s_{0}, s_{t}\right) .
\end{aligned}
$$

Theorem 13. Let $A=\left(s_{\theta}, s_{\sigma}\right)$ and $B=\left(s_{\mu}, s_{\nu}\right)$ be two LIFVs, where $s_{\theta}, s_{\sigma}, s_{\mu}, s_{v} \in \bar{S}=\left\{s_{\alpha} \mid s_{0}<s_{\alpha} \leq s_{t}, \alpha \in[0, t]\right\}$ with $\lambda, \lambda_{1}, \lambda_{2}>0$. Then, one has

(1) $\lambda(A \oplus B)=\lambda A \oplus \lambda B$,

(2) $\lambda_{1} A \oplus \lambda_{2} A=\left(\lambda_{1}+\lambda_{2}\right) A$,

(3) $A^{\lambda} \otimes B^{\lambda}=(A \otimes B)^{\lambda}$,

(4) $A^{\lambda_{1}} \otimes A^{\lambda_{2}}=A^{\lambda_{1}+\lambda_{2}}$.

Proof. (1) By (11), we have $A \oplus B=\left(s_{\theta+\mu-\theta \mu / t}, s_{\sigma \nu / t}\right)$. Thus, based on (13), we have

$$
\begin{aligned}
\lambda(A \oplus B) & =\left(s_{t\left(1-(1-(\theta+\mu-\theta \mu / t) / t)^{\lambda}\right)}, s_{t((\sigma v / t) / t)^{\lambda}}\right) \\
& =\left(s_{t\left(1-(1-\theta / t)^{\lambda}(1-\mu / t)^{\lambda}\right)}, s_{t(\sigma / t)^{\lambda}(v / t)^{\lambda}}\right) .
\end{aligned}
$$

Similarly, since $\lambda A=\left(s_{t\left(1-(1-\theta / t)^{\lambda}\right)}, s_{t(\sigma / t)^{\lambda}}\right)$ and $\lambda B=$ $\left(s_{t\left(1-(1-\mu / t)^{\lambda}\right)}, s_{t(v / t)^{\lambda}}\right)$, then

$\lambda A \oplus \lambda B$

$$
\begin{aligned}
& =\left(s_{t\left(1-(1-\theta / t)^{\lambda}\right)+t\left(1-(1-\mu / t)^{\lambda}\right)-t\left(1-(1-\theta / t)^{\lambda}\right)\left(1-(1-\mu / t)^{\lambda}\right),},\right. \\
& \left.s_{t(\sigma / t)^{\lambda} t(v / t)^{\lambda} / t}\right) \\
& =\left(s_{t\left(1-(1-\theta / t)^{\lambda}(1-\mu / t)^{\lambda}\right)}, s_{t(\sigma / t)^{\lambda}(v / t)^{\lambda}}\right) .
\end{aligned}
$$

Hence, we obtain $\lambda(A \oplus B)=\lambda A \oplus \lambda B$.

(2) By (13), we have $\lambda_{1} A=\left(s_{t\left(1-(1-\theta / t)^{\lambda_{1}}\right)}, s_{t(\sigma / t)^{\lambda_{1}}}\right)$ and $\lambda_{2} A=\left(s_{t\left(1-(1-\theta / t)^{\lambda_{2}}\right.}, s_{t(\sigma / t)^{\lambda_{2}}}\right)$; thus, we obtain

$$
\begin{aligned}
& \lambda_{1} A \oplus \lambda_{2} A \\
& =\left(s_{t\left(1-(1-\theta / t)^{\lambda_{1}}\right)+t\left(1-(1-\theta / t)^{\lambda_{2}}\right)-t\left(1-(1-\theta / t)^{\lambda_{1}}\right)\left(1-(1-\theta / t)^{\lambda_{2}}\right)},\right. \\
& \left.s_{t(\sigma / t)^{\lambda_{1}}(\sigma / t)^{\lambda_{2}}}\right) \\
& =\left(s_{t(1-\theta / t)^{\lambda_{1}}(1-\theta / t)^{\lambda_{2}}}, s_{t(\sigma / t)^{\lambda_{1}+\lambda_{2}}}\right) \\
& =\left(s_{t(1-\theta / t)^{\lambda_{1}+\lambda_{2}}}, s_{t(\sigma / t)^{\lambda_{1}+\lambda_{2}}}\right)=\left(\lambda_{1}+\lambda_{2}\right) A .
\end{aligned}
$$

(3) By (14), we get $A^{\lambda}=\left(s_{t(\theta / t)^{\lambda}}, s_{t\left(1-(1-\sigma / t)^{\lambda}\right)}\right)$ and $B^{\lambda}=$ $\left(s_{t(\mu / t)^{\lambda}}, s_{t\left(1-(1-v / t)^{\lambda}\right)}\right)$; thus, based on (12), we have

$A^{\lambda} \otimes B^{\lambda}$

$=\left(s_{t(\theta / t)^{\lambda}}(\mu / t)^{\lambda}, s_{t\left(1-(1-\sigma / t)^{\lambda}\right)+t\left(1-(1-v / t)^{\lambda}\right)-t\left(1-(1-\sigma / t)^{\lambda}\right)\left(1-(1-v / t)^{\lambda}\right)}\right)$

$=\left(s_{t(\theta / t)^{\lambda}(\mu / t)^{\lambda}}, s_{t\left(1-(1-\sigma / t)^{\lambda}(1-\nu / t)^{\lambda}\right)}\right)$. 
Since $A \otimes B=\left(s_{\theta \mu / t}, s_{\sigma+\nu-\sigma \nu / t}\right)$, then, by (14), we have

$$
\begin{aligned}
(A & \otimes B)^{\lambda} \\
& =\left(s_{t(\theta \mu / t / t)^{\lambda}}, s_{t\left(1-(1-(\sigma+\nu-\sigma \nu / t) / t)^{\lambda}\right)}\right) \\
& =\left(s_{t(\theta / t)^{\lambda}(\mu / t)^{\lambda}}, s_{t\left(1-\left(1-\sigma / t-v / t-\sigma \nu / t^{2}\right)^{\lambda}\right)}\right) \\
& =\left(s_{t(\theta / t)^{\lambda}(\mu / t)^{\lambda}}, s_{t\left(1-(1-\sigma / t)^{\lambda}(1-v / t)^{\lambda}\right)}\right) .
\end{aligned}
$$

Hence, we obtain $A^{\lambda} \otimes B^{\lambda}=(A \otimes B)^{\lambda}$.

(4) By (14), we have $A^{\lambda_{1}}=\left(s_{t(\theta / t)^{\lambda_{1}}}, s_{t\left(1-(1-\sigma / t)^{\lambda_{1}}\right)}\right)$ and $A^{\lambda_{2}}=\left(s_{t(\theta / t)^{\lambda_{2}}}, s_{t\left(1-(1-\sigma / t)^{\lambda_{2}}\right)}\right)$; thus, we have

$$
\begin{aligned}
A^{\lambda_{1}} \otimes & A^{\lambda_{2}} \\
= & \left(s_{t(\theta / t)^{\lambda_{1}}(\theta / t)^{\lambda_{2}},}\right. \\
& \left.\quad s_{t\left(1-(1-\sigma / t)^{\lambda_{1}}\right)+t\left(1-(1-\sigma / t)^{\lambda_{2}}\right)-t\left(1-(1-\sigma / t)^{\lambda_{1}}\right)\left(1-(1-\sigma / t)^{\lambda_{2}}\right)}\right) \\
= & \left(s_{\left.t(\theta / t)^{\lambda_{1}+\lambda_{2}}, s_{\left.t\left(1-(1-\sigma / t)^{\lambda_{1}}\right)(1-\sigma / t)^{\lambda_{2}}\right)}\right)}\right. \\
= & \left(s_{t(\theta / t)^{\lambda_{1}+\lambda_{2}},} s_{t\left(1-(1-\sigma / t)^{\lambda_{1}+\lambda_{2}}\right)}\right)=A^{\lambda_{1}+\lambda_{2}},
\end{aligned}
$$

which completes the proof of Theorem 13.

Motivated by the intuitionistic fuzzy aggregation operators $[14,15]$, in what follows, we define some aggregation operators for LIFVs.

Definition 14. Let $A_{i}=\left(s_{\theta_{i}}, s_{\sigma_{i}}\right)(i=1,2, \ldots, n)$ be a set of LIFVs. Then, the linguistic intuitionistic fuzzy weighted averaging (LIFWA) operator is defined as

$$
\begin{aligned}
\operatorname{LIFWA}\left(A_{1}, A_{2}, \ldots, A_{n}\right) & =w_{1} A_{1} \oplus w_{2} A_{2} \oplus \cdots \oplus w_{n} A_{n} \\
& =\left(s_{t\left(1-\prod_{i=1}^{n}\left(1-\theta_{i} / t\right)^{w_{i}}\right)}, s_{t \prod_{i=1}^{n}\left(\sigma_{i} / t\right)^{w_{i}}}\right),
\end{aligned}
$$

where $w=\left(w_{1}, w_{2}, \ldots, w_{n}\right)^{T}$ is the weight vector of $A_{i}(i=$ $1,2, \ldots, n)$, with $w_{i} \in[0,1]$ and $\sum_{i=1}^{n} w_{i}=1$.

In Particular, if $w=(1 / n, 1 / n, \ldots, 1 / n)^{T}$, then the LIFWA operator is reduced to a linguistic intuitionistic fuzzy averaging (LIFA) operator; that is,

$$
\begin{aligned}
\operatorname{LIFWA}\left(A_{1}, A_{2}, \ldots, A_{n}\right) & =\frac{1}{n} A_{1} \oplus \frac{1}{n} A_{2} \oplus \cdots \oplus \frac{1}{n} A_{n} \\
& =\left(s_{t\left(1-\prod_{i=1}^{n}\left(1-\theta_{i} / t\right)^{1 / n}\right)}, s_{t \prod_{i=1}^{n}\left(\sigma_{i} / t\right)^{1 / n}}\right) .
\end{aligned}
$$

Based on Definition 14, we get some properties of the LIFWA operator.

Theorem 15. Let $A_{i}=\left(s_{\theta_{i}}, s_{\sigma_{i}}\right)(i=1,2, \ldots, n)$ be a set of LIFVs and $w=\left(w_{1}, w_{2}, \ldots, w_{n}\right)^{T}$ the weight vector of $A_{i}(i=$ $0,1,2, \ldots, n)$, with $w_{i} \in[0,1]$ and $\sum_{i=1}^{n} w_{i}=1$; then one has the following.
(1) Idempotency. If all $A_{i}(i=1,2, \ldots, n)$ are equal, that is, $A_{i}=\left(s_{\theta_{i}}, s_{\sigma_{i}}\right)=\left(s_{\theta}, s_{\sigma}\right)$, for any $i$, then

$$
\operatorname{LIFWA}\left(A_{1}, A_{2}, \ldots, A_{n}\right)=\left(s_{\theta}, s_{\sigma}\right) .
$$

(2) Monotonicity. Let $A_{i}^{\prime}=\left(s_{\theta_{i}^{\prime}}, s_{\sigma_{i}^{\prime}}\right)(i=1,2, \ldots, n)$ be a set of LIFVs. If $s_{\theta_{i}^{\prime}} \geq s_{\theta_{i}}$ and $s_{\sigma_{i}^{\prime}} \leq s_{\sigma_{i}}$, for any $i$, then

$$
\operatorname{LIFWA}\left(A_{1}^{\prime}, A_{2}^{\prime}, \ldots, A_{n}^{\prime}\right) \geq \operatorname{LIFWA}\left(A_{1}, A_{2}, \ldots, A_{n}\right) \text {, }
$$

for any $w$.

(3) Boundary. Consider

$$
\begin{aligned}
\left(\min _{i}\left(s_{\theta_{i}}\right), \max _{i}\left(s_{\sigma_{i}}\right)\right) & \leq \operatorname{LIFWA}\left(A_{1}, A_{2}, \ldots, A_{n}\right) \\
& \leq\left(\max _{i}\left(s_{\theta_{i}}\right), \min _{i}\left(s_{\sigma_{i}}\right)\right) .
\end{aligned}
$$

Proof. (1) Since $A_{i}=\left(s_{\theta_{i}}, s_{\sigma_{i}}\right)=\left(s_{\theta}, s_{\sigma}\right)$, for any $i$, then

$$
\begin{aligned}
& \operatorname{LIFWA}\left(A_{1}, A_{2}, \ldots, A_{n}\right) \\
& =\left(s_{t\left(1-\prod_{i=1}^{n}(1-\theta / t)^{w_{i}}\right)}, s_{t \prod_{i=1}^{n}(\sigma / t)^{w_{i}}}\right) \\
& =\left(s_{t(1-(1-\theta / t))}, s_{t(\sigma / t)}\right)=\left(s_{\theta}, s_{\sigma}\right) .
\end{aligned}
$$

(2) If $s_{\theta_{i}^{\prime}} \geq s_{\theta_{i}}$, that is, $\theta_{i}^{\prime} \geq \theta_{i}$, for any $i$, then we have

$$
\begin{aligned}
\theta_{i}^{\prime} \geq \theta_{i} & \Longrightarrow 0 \leq 1-\frac{\theta_{i}^{\prime}}{t} \leq 1-\frac{\theta_{i}}{t} \leq 1 \\
& \Longrightarrow\left(1-\frac{\theta_{i}^{\prime}}{t}\right)^{w_{i}} \leq\left(1-\frac{\theta_{i}}{t}\right)^{w_{i}} \\
& \Longrightarrow \prod_{i=1}^{n}\left(1-\frac{\theta_{i}^{\prime}}{t}\right)^{w_{i}} \leq \prod_{i=1}^{n}\left(1-\frac{\theta_{i}}{t}\right)^{w_{i}} \\
& \Longrightarrow 1-\prod_{i=1}^{n}\left(1-\frac{\theta_{i}^{\prime}}{t}\right)^{w_{i}} \geq 1-\prod_{i=1}^{n}\left(1-\frac{\theta_{i}}{t}\right)^{w_{i}} \\
& \Longrightarrow t\left(1-\prod_{i=1}^{n}\left(1-\frac{\theta_{i}^{\prime}}{t}\right)^{w_{i}}\right) \geq t\left(1-\prod_{i=1}^{n}\left(1-\frac{\theta_{i}}{t}\right)^{w_{i}}\right) .
\end{aligned}
$$

Similarly, when $s_{\sigma_{i}^{\prime}} \leq s_{\sigma_{i}}$, for any $i$, we can get $t \prod_{i=1}^{n}\left(\sigma_{i}^{\prime} / t\right)^{1 / n} \leq t \prod_{i=1}^{n}\left(\sigma_{i} / t\right)^{1 / n}$.

According to Definition 7, we obtain

$$
\begin{aligned}
& \left(s_{t\left(1-\prod_{i=1}^{n}\left(1-\theta_{i}^{\prime} / t\right)^{1 / n}\right)}, s_{t \prod_{i=1}^{n}\left(\sigma_{i}^{\prime} / t\right)^{1 / n}}\right) \\
& \quad \geq\left(s_{t\left(1-\prod_{i=1}^{n}\left(1-\theta_{i} / t\right)^{1 / n}\right)}, s_{t \prod_{i=1}^{n}\left(\sigma_{i} / t\right)^{1 / n}}\right) ;
\end{aligned}
$$

that is,

$$
\operatorname{LIFWA}\left(A_{1}^{\prime}, A_{2}^{\prime}, \ldots, A_{n}^{\prime}\right) \geq \operatorname{LIFWA}\left(A_{1}, A_{2}, \ldots, A_{n}\right) \text {. }
$$


(3) Since $\min _{i}\left(s_{\theta_{i}}\right) \leq s_{\theta_{i}} \leq \max _{i}\left(s_{\theta_{i}}\right)$ and $\max _{i}\left(s_{\sigma_{i}}\right) \leq$ $s_{\sigma_{i}} \leq \min _{i}\left(s_{\sigma_{i}}\right)$, for any $i$, then, based on the monotonicity of Theorem 15, we derive

$$
\begin{aligned}
\left(\min _{i}\left(s_{\theta_{i}}\right), \max _{i}\left(s_{\sigma_{i}}\right)\right) & \leq \operatorname{LIFWA}\left(A_{1}, A_{2}, \ldots, A_{n}\right) \\
& \leq\left(\max _{i}\left(s_{\theta_{i}}\right), \min _{i}\left(s_{\sigma_{i}}\right)\right) .
\end{aligned}
$$

Definition 16. Let $A_{i}=\left(s_{\theta_{i}}, s_{\sigma_{i}}\right)(i=1,2, \ldots, n)$ be a set of LIFVs. Then, the linguistic intuitionistic fuzzy ordered weighted averaging (LIFOWA) operator is defined as

$$
\begin{aligned}
& \operatorname{LIFOWA}\left(A_{1}, A_{2}, \ldots, A_{n}\right) \\
& \quad=w_{1} A_{(1)} \oplus w_{2} A_{(2)} \oplus \cdots \oplus w_{n} A_{(n)} \\
& =\left(s_{t\left(1-\prod_{i=1}^{n}\left(1-\theta_{(i)} / t\right)^{w_{i}}\right)}, s_{t \prod_{i=1}^{n}\left(\sigma_{(i)} / t\right)^{w_{i}}}\right),
\end{aligned}
$$

where $A_{(i)}=\left(s_{\theta_{(i)}}, s_{\sigma_{(i)}}\right)$ is the $i$ th largest of $A_{1}, A_{2}, \ldots, A_{n}$ and $w=\left(w_{1}, w_{2}, \ldots, w_{n}\right)^{T}$ is the associated weight vector of $A_{(i)}(i=1,2, \ldots, n)$, with $w_{i} \in[0,1]$ and $\sum_{i=1}^{n} w_{i}=1$.

Similar to Theorem 15, we have some properties of the LIFOWA operator.

Theorem 17. Let $A_{i}=\left(s_{\theta_{i}}, s_{\sigma_{i}}\right)(i=1,2, \ldots, n)$ be a set of linguistic intuitionistic fuzzy values and $w=\left(w_{1}, w_{2}, \ldots, w_{n}\right)^{T}$ the associated weight vector of $A_{(i)}(i=1,2, \ldots, n)$, with $w_{i} \in$ $[0,1]$ and $\sum_{i=1}^{n} w_{i}=1$; then one has the following.

(1) Idempotency. If all $A_{i}(i=1,2, \ldots, n)$ are equal, that is, $A_{i}=\left(s_{\theta_{i}}, s_{\sigma_{i}}\right)=\left(s_{\theta}, s_{\sigma}\right)$, for any $i$, then

$$
\operatorname{LIFOWA}\left(A_{1}, A_{2}, \ldots, A_{n}\right)=\left(s_{\theta}, s_{\sigma}\right) \text {. }
$$

(2) Monotonicity. Let $A_{i}^{\prime}=\left(s_{\theta_{i}^{\prime}}, s_{\sigma_{i}^{\prime}}\right)(i=1,2, \ldots, n)$ be a set of linguistic intuitionistic fuzzy values. If $s_{\theta_{i}^{\prime}} \geq s_{\theta_{i}}$ and $s_{\sigma_{i}^{\prime}} \leq s_{\sigma_{i}}$, for any $i$, then

$$
\operatorname{LIFOWA}\left(A_{1}^{\prime}, A_{2}^{\prime}, \ldots, A_{n}^{\prime}\right) \geq \operatorname{LIFOWA}\left(A_{1}, A_{2}, \ldots, A_{n}\right) \text {, }
$$

for any $w$.

(3) Boundary. Consider

$$
\begin{aligned}
\left(\min _{i}\left(s_{\theta_{i}}\right), \max _{i}\left(s_{\sigma_{i}}\right)\right) & \leq \operatorname{LIFOWA}\left(A_{1}, A_{2}, \ldots, A_{n}\right) \\
& \leq\left(\max _{i}\left(s_{\theta_{i}}\right), \min _{i}\left(s_{\sigma_{i}}\right)\right) .
\end{aligned}
$$

(4) Commutativity. Let $A_{i}^{\prime}=\left(s_{\theta_{i}^{\prime}}, s_{\sigma_{i}^{\prime}}\right)(i=0,1,2, \ldots, n)$ be a set of linguistic intuitionistic fuzzy values, then for any $w$

$$
\operatorname{LIFOWA}\left(A_{1}^{\prime}, A_{2}^{\prime}, \ldots, A_{n}^{\prime}\right)=\operatorname{LIFOWA}\left(A_{1}, A_{2}, \ldots, A_{n}\right) \text {, }
$$

where $\left(A_{1}^{\prime}, A_{2}^{\prime}, \ldots, A_{n}^{\prime}\right)$ is any permutation of $\left(A_{1}, A_{2}, \ldots, A_{n}\right)$.

Definition 18. Let $A_{i}=\left(s_{\theta_{i}}, s_{\sigma_{i}}\right)(i=1,2, \ldots, n)$ be a set of LIFVs. Then, the linguistic intuitionistic fuzzy weighted geometric (LIFWG) operator is defined as

$$
\begin{aligned}
\operatorname{LIFWG}\left(A_{1}, A_{2}, \ldots, A_{n}\right) & =A_{1}^{w_{1}} \otimes A_{2}^{w_{2}} \otimes \cdots \otimes A_{n}^{w_{n}} \\
& =\left(s_{t \prod_{i=1}^{n}\left(\theta_{i} / t\right)^{w_{i}}}, s_{t\left(1-\prod_{i=1}^{n}\left(1-\sigma_{i} / t\right)^{w_{i}}\right)}\right),
\end{aligned}
$$

where $w=\left(w_{1}, w_{2}, \ldots, w_{n}\right)^{T}$ is the weight vector of $A_{i}(i=$ $1,2, \ldots, n)$, with $w_{i} \in[0,1]$ and $\sum_{i=1}^{n} w_{i}=1$.

The LIFWG operator has the following properties.

Theorem 19. Let $A_{i}=\left(s_{\theta_{i}}, s_{\sigma_{i}}\right)(i=1,2, \ldots, n)$ be a set of LIFVs and $w=\left(w_{1}, w_{2}, \ldots, w_{n}\right)^{T}$ the weight vector of $A_{i}(i=$ $1,2, \ldots, n)$, with $w_{i} \in[0,1]$ and $\sum_{i=1}^{n} w_{i}=1$; then one has the following.

(1) Idempotency. If all $A_{i}(i=1,2, \ldots, n)$ are equal, that is, $A_{i}=\left(s_{\theta_{i}}, s_{\sigma_{i}}\right)=\left(s_{\theta}, s_{\sigma}\right)$, for any $i$, then

$$
\operatorname{LIFWG}\left(A_{1}, A_{2}, \ldots, A_{n}\right)=\left(s_{\theta}, s_{\sigma}\right) \text {. }
$$

(2) Monotonicity. Let $A_{i}^{\prime}=\left(s_{\theta_{i}^{\prime}}, s_{\sigma_{i}^{\prime}}\right)(i=1,2, \ldots, n)$ be a set of linguistic intuitionistic fuzzy values. If $s_{\theta_{i}^{\prime}} \geq s_{\theta_{i}}$ and $s_{\sigma_{i}^{\prime}} \leq s_{\sigma_{i}}$, for any $i$, then

$$
\operatorname{LIFWG}\left(A_{1}^{\prime}, A_{2}^{\prime}, \ldots, A_{n}^{\prime}\right) \geq \operatorname{LIFWG}\left(A_{1}, A_{2}, \ldots, A_{n}\right) \text {, }
$$

for any $w$.

(3) Boundary. Consider

$$
\begin{aligned}
\left(\min _{i}\left(s_{\theta_{i}}\right), \max _{i}\left(s_{\sigma_{i}}\right)\right) & \leq \operatorname{LIFWG}\left(A_{1}, A_{2}, \ldots, A_{n}\right) \\
& \leq\left(\max _{i}\left(s_{\theta_{i}}\right), \min _{i}\left(s_{\sigma_{i}}\right)\right) .
\end{aligned}
$$

Definition 20. Let $A_{i}=\left(s_{\theta_{i}}, s_{\sigma_{i}}\right)(i=1,2, \ldots, n)$ be a set of LIFVs. Then, the linguistic intuitionistic fuzzy ordered weighted geometric (LIFOWG) operator is defined as

$$
\begin{aligned}
& \operatorname{LIFOWG}\left(A_{1}, A_{2}, \ldots, A_{n}\right) \\
& \quad=A_{(1)}^{w_{1}} \otimes A_{(2)}^{w_{2}} \otimes \cdots \otimes A_{(n)}^{w_{n}} \\
& =\left(s_{t \prod_{i=1}^{n}\left(\theta_{(i)} / t\right)^{w_{i}}}, s_{t\left(1-\prod_{i=1}^{n}\left(1-\sigma_{(i)} / t\right)^{w_{i}}\right)}\right),
\end{aligned}
$$

where $A_{(i)}=\left(s_{\theta_{(i)}}, s_{\sigma_{(i)}}\right)$ is the $i$ th largest of $A_{1}, A_{2}, \ldots, A_{n}$ and $w=\left(w_{1}, w_{2}, \ldots, w_{n}\right)^{T}$ is the associated weight vector of $A_{(i)}(i=1,2, \ldots, n)$, with $w_{i} \in[0,1]$ and $\sum_{i=1}^{n} w_{i}=1$.

Similar to Theorem 15, we have some properties of the LIFOWG operator. 
Theorem 21. Let $A_{i}=\left(s_{\theta_{i}}, s_{\sigma_{i}}\right)(i=1,2, \ldots, n)$ be a set of LIFVs and $w=\left(w_{1}, w_{2}, \ldots, w_{n}\right)^{T}$ the associated weight vector of $A_{(i)}(i=1,2, \ldots, n)$, with $w_{i} \in[0,1]$ and $\sum_{i=1}^{n} w_{i}=1$; then one has the following.

(1) Idempotency. If all $A_{i}(i=1,2, \ldots, n)$ are equal, that is, $A_{i}=\left(s_{\theta_{i}}, s_{\sigma_{i}}\right)=\left(s_{\theta}, s_{\sigma}\right)$, for any $i$, then

$$
\operatorname{LIFOWG}\left(A_{1}, A_{2}, \ldots, A_{n}\right)=\left(s_{\theta}, s_{\sigma}\right) .
$$

(2) Monotonicity. Let $A_{i}^{\prime}=\left(s_{\theta_{i}^{\prime}}, s_{\sigma_{i}^{\prime}}\right)(i=1,2, \ldots, n)$ be a set of linguistic intuitionistic fuzzy values. If $s_{\theta_{i}^{\prime}} \geq s_{\theta_{i}}$ and $s_{\sigma_{i}^{\prime}} \leq s_{\sigma_{i}}$, for any $i$, then

$$
\operatorname{LIFOWG}\left(A_{1}^{\prime}, A_{2}^{\prime}, \ldots, A_{n}^{\prime}\right) \geq \operatorname{LIFOWG}\left(A_{1}, A_{2}, \ldots, A_{n}\right) \text {, }
$$

for any $w$.

(3) Boundary. Consider

$$
\begin{aligned}
\left(\min _{i}\left(s_{\theta_{i}}\right), \max _{i}\left(s_{\sigma_{i}}\right)\right) & \leq \operatorname{LIFOWG}\left(A_{1}, A_{2}, \ldots, A_{n}\right) \\
& \leq\left(\max _{i}\left(s_{\theta_{i}}\right), \min _{i}\left(s_{\sigma_{i}}\right)\right) .
\end{aligned}
$$

(4) Commutativity. Let $A_{i}^{\prime}=\left(s_{\theta_{i}^{\prime}}, s_{\sigma_{i}^{\prime}}\right)(i=1,2, \ldots, n)$ be a set of linguistic intuitionistic fuzzy values; then for any $w$

$$
\operatorname{LIFOWG}\left(A_{1}^{\prime}, A_{2}^{\prime}, \ldots, A_{n}^{\prime}\right)=\operatorname{LIFOWG}\left(A_{1}, A_{2}, \ldots, A_{n}\right) \text {, }
$$

where $\left(A_{1}^{\prime}, A_{2}^{\prime}, \ldots, A_{n}^{\prime}\right)$ is any permutation of $\left(A_{1}, A_{2}, \ldots, A_{n}\right)$.

Lemma 22 (see $[37,38])$. Let $x_{i}>0, \lambda_{i}>0, i=1,2, \ldots, n$, and $\sum_{i=1}^{n} \lambda_{i}=1$; then

$$
\prod_{i=1}^{n} x_{i}^{\lambda_{i}} \leq \sum_{i=1}^{n} \lambda_{i} x_{i}
$$

with equality if and only if $x_{1}=x_{2}=\cdots=x_{n}$.

Based on Lemma 22, we have the following theorem.

Theorem 23. Let $A_{i}=\left(s_{\theta_{i}}, s_{\sigma_{i}}\right)(i=1,2, \ldots, n)$ be a set of LIFVs; then one has

$$
\begin{gathered}
\operatorname{LIFWA}\left(A_{1}, A_{2}, \ldots, A_{n}\right) \geq \operatorname{LIFWG}\left(A_{1}, A_{2}, \ldots, A_{n}\right), \\
\operatorname{LIFOWA}\left(A_{1}, A_{2}, \ldots, A_{n}\right) \geq \operatorname{LIFOWG}\left(A_{1}, A_{2}, \ldots, A_{n}\right),
\end{gathered}
$$

with equality if and only if $A_{1}=A_{2}=\cdots=A_{n}$.

Proof. Let $w=\left(w_{1}, w_{2}, \ldots, w_{n}\right)^{T}$ be the weight vector of $A_{i}(i=1,2, \ldots, n)$, with $w_{i} \in[0,1]$ and $\sum_{i=1}^{n} w_{i}=1$; then, by Lemma 22 , we have $1-\prod_{i=1}^{n}\left(1-\theta_{i} / t\right)^{w_{i}} \geq 1-\sum_{i=1}^{n} w_{i}(1-$ $\left.\theta_{i} / t\right)=1-\sum_{i=1}^{n} w_{i}+\sum_{i=1}^{n} w_{i} \theta_{i} / t=\sum_{i=1}^{n} w_{i} \theta_{i} / t \geq \prod_{i=1}^{n}\left(\theta_{i} / t\right)^{w_{i}}$, with equality if and only if $\theta_{1}=\theta_{2}=\cdots=\theta_{n}$; that is, $t\left(1-\prod_{i=1}^{n}\left(1-\theta_{i} / t\right)^{w_{i}}\right) \geq t \prod_{i=1}^{n}\left(\theta_{i} / t\right)^{w_{i}}$, with equality if and only if $\theta_{1}=\theta_{2}=\cdots=\theta_{n}$; and $\prod_{i=1}^{n}\left(\sigma_{i} / t\right)^{w_{i}} \leq \sum_{i=1}^{n} w_{i} \sigma_{i} / t=$ $1-\sum_{i=1}^{n} w_{i}\left(1-\sigma_{i} / t\right) \leq 1-\prod_{i=1}^{n}\left(1-\sigma_{i} / t\right)^{w_{i}}$, with equality if and only if $\sigma_{1}=\sigma_{2}=\cdots=\sigma_{n}$; that is, $t\left(\prod_{i=1}^{n}\left(\sigma_{i} / t\right)^{w_{i}}\right) \leq$ $t\left(1-\prod_{i=1}^{n}\left(1-\sigma_{i} / t\right)^{w_{i}}\right)$, with equality if and only if $\sigma_{1}=\sigma_{2}=$ $\cdots=\sigma_{n}$.

Consequently, by Definition 7, we obtain $\left(s_{t\left(1-\prod_{i=1}^{n}\left(1-\theta_{i} / t\right)^{w_{i}}\right)}, s_{t \prod_{i=1}^{n}\left(\sigma_{i} / t\right)^{w_{i}}}\right) \quad \geq \quad\left(s_{t \prod_{i=1}^{n}\left(\theta_{i} / t\right)^{w_{i}}}\right.$, $\left.s_{t\left(1-\prod_{i=1}^{n}\left(1-\sigma_{i} / t\right)^{w_{i}}\right)}\right)$, with equality if and only if $\theta_{1}=\theta_{2}=$ $\cdots=\theta_{n}$ and $\sigma_{1}=\sigma_{2}=\cdots=\sigma_{n}$; that is,

$$
\operatorname{LIFWA}\left(A_{1}, A_{2}, \ldots, A_{n}\right) \geq \operatorname{LIFWG}\left(A_{1}, A_{2}, \ldots, A_{n}\right)
$$

with equality if and only if $A_{1}=A_{2}=\cdots=A_{n}$.

Similarly, we can also prove $\operatorname{LIFOWA}\left(A_{1}, A_{2}, \ldots, A_{n}\right) \geq$ $\operatorname{LIFOWG}\left(A_{1}, A_{2}, \ldots, A_{n}\right)$, with equality if and only if $A_{1}=$ $A_{2}=\cdots=A_{n}$.

Besides the above properties, we can derive the following desirable results of the LIFOWA and LIFOWG operators.

Theorem 24. Let $A_{i}=\left(s_{\theta_{i}}, s_{\sigma_{i}}\right)(i=1,2, \ldots, n)$ be a set of LIFVs and $w=\left(w_{1}, w_{2}, \ldots, w_{n}\right)^{T}$ the associated weight vector of $A_{(i)}(i=1,2, \ldots, n)$, with $w_{i} \in[0,1]$ and $\sum_{i=1}^{n} w_{i}=1$. Then one has the following.

(1) If $w=(1,0, \ldots, 0)^{T}$, then

$$
\begin{aligned}
\operatorname{LIFOWA}\left(A_{1}, A_{2}, \ldots, A_{n}\right) & =\operatorname{LIFOWG}\left(A_{1}, A_{2}, \ldots, A_{n}\right) \\
& =\max _{i}\left(A_{i}\right)
\end{aligned}
$$

(2) If $w=(0,0, \ldots, 1)^{T}$, then

$$
\begin{aligned}
\operatorname{LIFOWA}\left(A_{1}, A_{2}, \ldots, A_{n}\right) & =\operatorname{LIFOWG}\left(A_{1}, A_{2}, \ldots, A_{n}\right) \\
& =\min _{i}\left(A_{i}\right)
\end{aligned}
$$

(3) If $w_{i}=1$ and $w_{j}=0$, for $j \neq i$, then

$$
\begin{aligned}
\operatorname{LIFOWA}\left(A_{1}, A_{2}, \ldots, A_{n}\right) & =\operatorname{LIFOWG}\left(A_{1}, A_{2}, \ldots, A_{n}\right) \\
& =A_{(i)}
\end{aligned}
$$

where $A_{(i)}=\left(s_{\theta_{(i)}}, s_{\sigma_{(i)}}\right)$ is the ith largest of $A_{1}, A_{2}, \ldots, A_{n}$.

Example 25. Let $A_{1}=\left(s_{1}, s_{2}\right), A_{2}=\left(s_{1}, s_{3}\right), A_{3}=\left(s_{2}, s_{4}\right)$, and $A_{4}=\left(s_{4}, s_{1}\right)$ be LIFVs, which are derived from $\bar{S}=\left\{s_{\alpha} \mid\right.$ $\left.s_{0}<s_{\alpha} \leq s_{6}, \alpha \in[0,6]\right\}$, and let $w=(0.2,0.3,0.4,0.1)^{T}$ be the weight vector of $A_{i}(i=1,2,3,4)$. 
Applying (26) and (41), we obtain the aggregated LIFVs as follows:

$$
\begin{aligned}
& \operatorname{LIFWA}\left(A_{1}, A_{2}, \ldots, A_{4}\right)=\left(s_{6\left(1-\prod_{i=1}^{4}\left(1-\theta_{i} / 6\right)^{w_{i}}\right)}, s_{6 \prod_{i=1}^{4}\left(\sigma_{i} / 6\right)^{w_{i}}}\right) \\
& =\left(s_{1.827}, s_{2.781}\right) \text {, } \\
& \operatorname{LIFWG}\left(A_{1}, A_{2}, \ldots, A_{4}\right)=\left(s_{6 \prod_{i=1}^{4}\left(\theta_{i} / 6\right)^{w_{i}}}, s_{6\left(1-\prod_{i=1}^{4}\left(1-\sigma_{i} / 6\right)^{w_{i}}\right)}\right) \\
& =\left(s_{1.516}, s_{3.157}\right) \text {. }
\end{aligned}
$$

It is easy to see that

$$
\begin{aligned}
\operatorname{LIFWA}\left(A_{1}, A_{2}, \ldots, A_{4}\right) & =\left(s_{1.827}, s_{2.781}\right) \\
& >\operatorname{LiFWG}\left(A_{1}, A_{2}, \ldots, A_{4}\right) \\
& =\left(s_{1.516}, s_{3.157}\right) .
\end{aligned}
$$

By Definition 7, we calculate the following values of score function and accuracy function.

$$
\begin{array}{cc}
S\left(A_{1}\right)=s_{(6+1-2) / 2}=s_{2.5}, & S\left(A_{2}\right)=s_{(6+1-3) / 2}=s_{2}, \\
S\left(A_{3}\right)=s_{(6+2-4) / 2}=s_{2}, & S\left(A_{4}\right)=s_{(6+4-1) / 2}=s_{4.5}, \\
H\left(A_{2}\right)=s_{1+3}=s_{4}, & H\left(A_{3}\right)=s_{2+4}=s_{6} .
\end{array}
$$

Since $S\left(A_{4}\right)>S\left(A_{1}\right)>S\left(A_{2}\right)=S\left(A_{3}\right)$ and $H\left(A_{3}\right)>$ $H\left(A_{2}\right)$, then

$$
\begin{array}{ll}
A_{(1)}=A_{4}=\left(s_{4}, s_{1}\right), & A_{(2)}=A_{1}=\left(s_{1}, s_{2}\right), \\
A_{(3)}=A_{3}=\left(s_{2}, s_{4}\right), & A_{(4)}=A_{2}=\left(s_{1}, s_{3}\right) .
\end{array}
$$

Assume that $w=(0.155,0.345,0.345,0.155)^{T}$, which are determined by the normal distribution based method [39], is the associated weight vector of $A_{(i)}$. Then, by (36) and (45), we have

$$
\begin{aligned}
& \operatorname{LIFOWA}\left(A_{1}, A_{2}, \ldots, A_{n}\right) \\
& =\left(s_{6\left(1-\prod_{i=1}^{4}\left(1-\theta_{(i)} / 6\right)^{w_{i}}\right)}, s_{6 \prod_{i=1}^{n}\left(\sigma_{(i)} / 6\right)^{w_{i}}}\right) \\
& \quad=\left(s_{1.983}, s_{2.430}\right), \\
& \operatorname{LIFOWG}\left(A_{1}, A_{2}, \ldots, A_{n}\right) \\
& =\left(s_{6 \prod_{i=1}^{n}\left(\theta_{(i)} / 6\right)^{w_{i}}}, s_{6\left(1-\prod_{i=1}^{n}\left(1-\sigma_{(i)} / 6\right)^{w_{i}}\right)}\right) \\
& =\left(s_{1.575}, s_{2.882}\right) .
\end{aligned}
$$

It is easy to see that

$$
\begin{aligned}
\operatorname{LifOWA}\left(A_{1}, A_{2}, \ldots, A_{4}\right) & =\left(s_{1.983}, s_{2.430}\right) \\
& >\operatorname{LiFOWG}\left(A_{1}, A_{2}, \ldots, A_{4}\right) \\
& =\left(s_{1.575}, s_{2.882}\right) .
\end{aligned}
$$

\section{MAGDM Method with Linguistic Intuitionistic Fuzzy Information}

In the following, we present a handling method for MAGDM problems, where the weight vector of attributes is known and the attribute performance values take the form of LIFVs.

Let $A=\left(A_{1}, A_{2}, \ldots, A_{m}\right)$ be the set of alternatives and $C=\left(C_{1}, C_{2}, \ldots, C_{n}\right)$ the set of attributes, whose weight vector is $w=\left(w_{1}, w_{2}, \ldots, w_{n}\right)$, with $w_{i} \in[0,1]$ and $\sum_{i=1}^{n} w_{i}=1$. Let $D=\left(D_{1}, D_{2}, \ldots, D_{l}\right)$ be the set of decision makers. Suppose $R_{k}=\left(r_{i j}^{k}\right)_{m \times n}$ is the decision matrix, where $r_{i j}^{k}=\left(s_{\theta_{i j}}^{k}, s_{\sigma_{i j}}^{k}\right)$ denotes the preference value and takes the form of LIFV, which is given by decision makers $D_{k}$ for alternative $A_{i}$ with respect to attribute $C_{j}$ and $s_{\theta_{i j}}^{k}, s_{\sigma_{i j}}^{k} \in S=\left\{s_{i} \mid i=0,1, \ldots, t\right\}$.

The proposed method is described as follows.

Step 1. Construct the linguistic intuitionistic fuzzy decision matrix $R_{k}=\left(r_{i j}^{k}\right)_{m \times n}$.

Step 2. Utilize the LIFOWA or LIFOWG operator to derive the aggregated decision matrix $R=\left(r_{i j}\right)_{m \times n}$ :

$$
\begin{gathered}
r_{i j}=\left(s_{\theta_{i j}}, s_{\sigma_{i j}}\right)=\operatorname{LIFOWA}\left(r_{i j}^{1}, r_{i j}^{2}, \ldots, r_{i j}^{l}\right), \\
\text { or } r_{i j}=\left(s_{\theta_{i j}}, s_{\sigma_{i j}}\right)=\operatorname{LIFOWG}\left(r_{i j}^{1}, r_{i j}^{2}, \ldots, r_{i j}^{l}\right),
\end{gathered}
$$

where the LIFOWA and LIFOWG weights are determined by the normal distribution based method [39].

Step 3. Aggregate $r_{i j}(j=1,2, \ldots, n)$ to yield the collective overall preference values $r_{i}$ for each alternative $A_{i} \quad(i=$ $1,2, \ldots, m)$ based on the LIFWA or LIFWG operator.

Step 4. Rank the alternatives in accordance with $r_{i}$, according to Definition 7.

\section{Numerical Example}

In this section, we consider an example adapted from Herrera and Herrera-Viedma [40]. Suppose an investment company, which wants to invest a sum of money in the best option. There is a panel with four possible alternatives of where to invest the money: $A_{1}$ is a car industry; $A_{2}$ is a food company; $A_{3}$ is a computer company; $A_{4}$ is an arms industry. The investment company must make a decision according to four criteria: $C_{1}$ is the risk analysis; $C_{2}$ is the growth analysis; $C_{3}$ is the social-political impact analysis; $C_{4}$ is the environmental impact analysis. The weight vector of attributes is $w=(0.3,0.1,0.2,0.4)$. Three experts are invited to provide their preferences for each alternative on each attribute with the linguistic term set $S=$ $\left\{s_{0}=\right.$ extremely poor, $s_{1}=$ very poor, $s_{2}=$ poor, $s_{3}=$ slightly poor, $s_{4}=$ fair, $s_{5}=$ slightly good, $s_{6}=$ good, $s_{7}=$ very good, $s_{8}=$ extremely good $\}$. 
TABLE 1: The decision matrix $R_{1}$ given by $D_{1}$.

\begin{tabular}{lcccc}
\hline & $C_{1}$ & $C_{2}$ & $C_{3}$ & $C_{4}$ \\
\hline$A_{1}$ & $\left(s_{6}, s_{1}\right)$ & $\left(s_{3}, s_{1}\right)$ & $\left(s_{3}, s_{3}\right)$ & $\left(s_{1}, s_{6}\right)$ \\
$A_{2}$ & $\left(s_{3}, s_{4}\right)$ & $\left(s_{3}, s_{4}\right)$ & $\left(s_{2}, s_{5}\right)$ & $\left(s_{2}, s_{4}\right)$ \\
$A_{3}$ & $\left(s_{1}, s_{3}\right)$ & $\left(s_{2}, s_{3}\right)$ & $\left(s_{3}, s_{2}\right)$ & $\left(s_{6}, s_{1}\right)$ \\
$A_{4}$ & $\left(s_{6}, s_{2}\right)$ & $\left(s_{4}, s_{3}\right)$ & $\left(s_{5}, s_{1}\right)$ & $\left(s_{7}, s_{1}\right)$ \\
\hline
\end{tabular}

TABle 2: The decision matrix $R_{2}$ given by $D_{2}$.

\begin{tabular}{lcccc}
\hline & $C_{1}$ & $C_{2}$ & $C_{3}$ & $C_{4}$ \\
\hline$A_{1}$ & $\left(s_{3}, s_{2}\right)$ & $\left(s_{4}, s_{1}\right)$ & $\left(s_{3}, s_{4}\right)$ & $\left(s_{2}, s_{3}\right)$ \\
$A_{2}$ & $\left(s_{5}, s_{2}\right)$ & $\left(s_{2}, s_{1}\right)$ & $\left(s_{3}, s_{4}\right)$ & $\left(s_{2}, s_{5}\right)$ \\
$A_{3}$ & $\left(s_{2}, s_{3}\right)$ & $\left(s_{3}, s_{3}\right)$ & $\left(s_{1}, s_{2}\right)$ & $\left(s_{3}, s_{3}\right)$ \\
$A_{4}$ & $\left(s_{5}, s_{2}\right)$ & $\left(s_{3}, s_{3}\right)$ & $\left(s_{5}, s_{2}\right)$ & $\left(s_{4}, s_{1}\right)$ \\
\hline
\end{tabular}

TABLE 3: The decision matrix $R_{3}$ given by $D_{3}$.

\begin{tabular}{lcccc}
\hline & $C_{1}$ & $C_{2}$ & $C_{3}$ & $C_{4}$ \\
\hline$A_{1}$ & $\left(s_{3}, s_{3}\right)$ & $\left(s_{3}, s_{5}\right)$ & $\left(s_{6}, s_{1}\right)$ & $\left(s_{2}, s_{6}\right)$ \\
$A_{2}$ & $\left(s_{3}, s_{2}\right)$ & $\left(s_{2}, s_{4}\right)$ & $\left(s_{2}, s_{1}\right)$ & $\left(s_{3}, s_{4}\right)$ \\
$A_{3}$ & $\left(s_{6}, s_{1}\right)$ & $\left(s_{2}, s_{5}\right)$ & $\left(s_{3}, s_{4}\right)$ & $\left(s_{1}, s_{3}\right)$ \\
$A_{4}$ & $\left(s_{5}, s_{1}\right)$ & $\left(s_{4}, s_{4}\right)$ & $\left(s_{6}, s_{2}\right)$ & $\left(s_{5}, s_{2}\right)$ \\
\hline
\end{tabular}

Step 1. The decision makers provide their evaluation values and construct the linguistic intuitionistic fuzzy decision matrix $R_{k}=\left(r_{i j}^{k}\right)_{m \times n}(k=1,2,3)$ as shown in Tables 1, 2, and 3, respectively.

Step 2. By the normal distribution based method [39], the associated weight vector is determined as $\omega=$ $(0.243,0.514,0.243)^{T}$. Then, utilize the LIFOWA operator to derive the aggregated decision matrix $R=\left(r_{i j}\right)_{4 \times 4}$, which is shown in Table 4. Alternatively, if the LIFOWG operator instead of LIFOWA operator is applied in Step 2, the aggregated decision matrix $R=\left(r_{i j}\right)_{m \times n}$ can be derived as shown in Table 5.

Step 3. Aggregate $r_{i j}(j=1,2, \ldots, 4)$ to yield the collective overall preference values $r_{i}$ for each alternative $A_{i}(i=$ $1,2, \ldots, 4)$ based on the LIFWA or LIFWG operator with the weight vector $w=(0.3,0.1,0.2,0.4)$, as shown in Table 6 .

Step 4. By ranking $r_{i}(i=1,2,3,4)$ based on Definition 7 , the priorities of the alternatives can be obtained, which are listed in Table 6. Obviously, both the methods come to the same conclusion that the best alternative is $A_{4}$.

In the above linguistic intuitionistic fuzzy aggregation operators, the weights of the arguments are supposed to be crisp numbers. However, for reason of complexity or uncertainty of decision making, decision makers may have difficulty in assigning weights of the attributes with crisp numbers and may be inclined to appoint the weights of the attributes as linguistic values, interval linguistic values, or LIFVs. The existing linguistic aggregation operators cannot deal with such cases. According to the operation laws
TABLE 4: The aggregated decision matrix $R$ by LIFOWA operator.

\begin{tabular}{ccccc}
\hline & $C_{1}$ & $C_{2}$ & $C_{3}$ & $C_{4}$ \\
\hline$A_{1}$ & $\left(s_{3.998}, s_{1.865}\right)$ & $\left(s_{3.264}, s_{1.479}\right)$ & $\left(s_{3.998}, s_{2.463}\right)$ & $\left(s_{1.771}, s_{5.070}\right)$ \\
$A_{2}$ & $\left(s_{3.584}, s_{2.367}\right)$ & $\left(s_{2.537}, s_{2.856}\right)$ & $\left(s_{2.537}, s_{3.051}\right)$ & $\left(s_{2.260}, s_{4.223}\right)$ \\
$A_{3}$ & $\left(s_{3.230}, s_{2.297}\right)$ & $\left(s_{2.260}, s_{3.397}\right)$ & $\left(s_{2.574}, s_{2.856}\right)$ & $\left(s_{3.657}, s_{2.297}\right)$ \\
$A_{4}$ & $\left(s_{5.282}, s_{1.401}\right)$ & $\left(s_{3.777}, s_{3.478}\right)$ & $\left(s_{5.282}, s_{1.401}\right)$ & $\left(s_{4.720}, s_{1.184}\right)$ \\
\hline
\end{tabular}

TABLE 5: The aggregated decision matrix $R$ by LIFOWG operator.

\begin{tabular}{ccccc}
\hline & $C_{1}$ & $C_{2}$ & $C_{3}$ & $C_{4}$ \\
\hline$A_{1}$ & $\left(s_{3.550}, s_{2.041}\right)$ & $\left(s_{3.217}, s_{2.303}\right)$ & $\left(s_{3.550}, s_{2.860}\right)$ & $\left(s_{1.690}, s_{5.501}\right)$ \\
$A_{2}$ & $\left(s_{3.397}, s_{2.563}\right)$ & $\left(s_{2.463}, s_{3.417}\right)$ & $\left(s_{2.462}, s_{3.727}\right)$ & $\left(s_{2.207}, s_{4.270}\right)$ \\
$A_{3}$ & $\left(s_{2.207}, s_{2.574}\right)$ & $\left(s_{2.207}, s_{3.584}\right)$ & $\left(s_{2.719}, s_{3.129}\right)$ & $\left(s_{2.719}, s_{2.774}\right)$ \\
$A_{4}$ & $\left(s_{5.227}, s_{1.505}\right)$ & $\left(s_{3.730}, s_{3.542}\right)$ & $\left(s_{5.227}, s_{1.505}\right)$ & $\left(s_{4.838}, s_{1.257}\right)$ \\
\hline
\end{tabular}

described by (11) and (12), we can also solve such decision making problems. For example, suppose the decision makers take the weights of attributes as linguistic values in this example, that is, $w_{1}=\left(s_{3}, s_{2}\right), w_{2}=s_{1}, w_{3}=\left[s_{2}, s_{4}\right]$, and $w_{4}=\left[s_{4}, s_{7}\right]$, which can be transformed to LIFVs; that is, $w_{1}=$ $\left(s_{3}, s_{2}\right), w_{2}=\left(s_{1}, s_{7}\right), w_{3}=\left(s_{2}, s_{4}\right)$, and $w_{4}=\left(s_{4}, s_{1}\right)$. Thus, by (11) and (12), the collective overall preference values $r_{i}$ for each alternative $A_{i}(i=1,2, \ldots, 4)$ with linguistic weights can be obtained by the following:

$$
\begin{aligned}
r_{i}= & \left(w_{1} \otimes r_{i 1}\right) \oplus\left(w_{2} \otimes r_{i 2}\right) \oplus\left(w_{3} \otimes r_{i 3}\right) \\
& \oplus\left(w_{4} \otimes r_{i 4}\right) .
\end{aligned}
$$

Without loss of generalization, on the basis of Table 4, we have the following results:

$$
\begin{aligned}
r_{1}= & \left(\left(s_{3}, s_{2}\right) \otimes\left(s_{3.998}, s_{1.865}\right)\right) \oplus\left(\left(s_{1}, s_{7}\right) \otimes\left(s_{3.264}, s_{1.479}\right)\right) \\
& \oplus\left(\left(s_{2}, s_{4}\right) \otimes\left(s_{3.998}, s_{2.463}\right)\right) \oplus\left(\left(s_{4}, s_{1}\right) \otimes\left(s_{1.771}, s_{5.070}\right)\right) \\
= & \left(s_{3.200}, s_{1.245}\right), \\
r_{2}= & \left(\left(s_{3}, s_{2}\right) \otimes\left(s_{3.584}, s_{2.367}\right)\right) \oplus\left(\left(s_{1}, s_{7}\right) \otimes\left(s_{2.537}, s_{2.856}\right)\right) \\
& \oplus\left(\left(s_{2}, s_{4}\right) \otimes\left(s_{2.537}, s_{3.051}\right)\right) \oplus\left(\left(s_{4}, s_{1}\right) \otimes\left(s_{2.260}, s_{4.223}\right)\right) \\
= & \left(s_{2.931}, s_{1.221}\right), \\
r_{3}= & \left(\left(s_{3}, s_{2}\right) \otimes\left(s_{3.230}, s_{2.297}\right)\right) \oplus\left(\left(s_{1}, s_{7}\right) \otimes\left(s_{2.260}, s_{3.397}\right)\right) \\
& \oplus\left(\left(s_{2}, s_{4}\right) \otimes\left(s_{2.574}, s_{2.856}\right)\right) \oplus\left(\left(s_{4}, s_{1}\right) \otimes\left(s_{3.657}, s_{2.297}\right)\right) \\
= & \left(s_{3.355}, s_{0.882}\right), \\
r_{4}= & \left(\left(s_{3}, s_{2}\right) \otimes\left(s_{5.282}, s_{1.401}\right)\right) \oplus\left(\left(s_{1}, s_{7}\right) \otimes\left(s_{3.777}, s_{3.478}\right)\right) \\
& \oplus\left(\left(s_{2}, s_{4}\right) \otimes\left(s_{5.282}, s_{1.401}\right)\right) \oplus\left(\left(s_{4}, s_{1}\right) \otimes\left(s_{4.720}, s_{1.184}\right)\right) \\
= & \left(s_{5.109}, s_{0.424}\right),
\end{aligned}
$$

by which we can obtain the rankings of alternatives: that is, $A_{4}>A_{3}>A_{1}>A_{2}$. 
TABLE 6: The collective overall preference values and the rankings of alternatives.

\begin{tabular}{lccccc}
\hline & $A_{1}$ & $A_{2}$ & $A_{3}$ & $A_{4}$ & Ranking \\
\hline LIFOWA & $\left(s_{3.142}, s_{2.874}\right)$ & $\left(s_{2.772}, s_{3.199}\right)$ & $\left(s_{3.198}, s_{2.495}\right)$ & $\left(s_{4.938}, s_{1.435}\right)$ & $A_{4}>A_{3}>A_{1}>A_{2}$ \\
LIFWA & $\left(s_{2.612}, s_{3.932}\right)$ & $\left(s_{2.596}, s_{3.619}\right)$ & $\left(s_{2.501}, s_{2.876}\right)$ & $\left(s_{4.990}, s_{1.650}\right)$ & $A_{4}>A_{3}>A_{2}>A_{1}$ \\
LIFOWG & &
\end{tabular}

Such results do not exceed the cardinality of the corresponding linguistic term set and can be easily accepted. By contrast, if we transform the above LIFVs into interval linguistic values and take the operation laws defined by Definition 3, we may have similar problems discussed in Example 11.

\section{Conclusions}

In this paper, we introduce the concept of LIFS, which can be seen as a generalization of IFS and is suitable to deal with imprecise and uncertain information in decision making. We further define the score function and accuracy function to compare the LIFVs. Moreover, we propose several aggregation operators for LIFSs, such as the LIFWA operator, LIFOWA operator, LIFWG operator, and LIFOWG operator, together with their desired properties. Comparing with the existing linguistic operation laws, we can obtain more intuitive and acceptable results by these aggregation operators proposed.

\section{Conflict of Interests}

The author declares that there is no conflict of interests regarding the publication of this paper.

\section{Acknowledgments}

The authors wish to thank the anonymous referees for their valuable comments and suggestions, which helped to improve this paper. This work was supported by the National Natural Science Foundation of China under Grant no. U1304701, the High-Level Talent Project of Henan University of Technology under Grant no. 2013BS015, and the Plan of Nature Science Fundamental Research in Henan University of Technology under Grant no. 2013JCYJ14.

\section{References}

[1] K. T. Atanassov, "Intuitionistic fuzzy sets," Fuzzy Sets and Systems, vol. 20, no. 1, pp. 87-96, 1986.

[2] P. Burillo and H. Bustince, "Entropy on intuitionistic fuzzy sets and on interval-valued fuzzy sets," Fuzzy Sets and Systems, vol. 78, no. 3, pp. 305-316, 1996.

[3] E. Szmidt and J. Kacprzyk, "Entropy for intuitionistic fuzzy sets," Fuzzy Sets and Systems, vol. 118, no. 3, pp. 467-477, 2001.

[4] H. M. Zhang, "Entropy for intuitionistic fuzzy set based on distance and intuitionistic index," International Journal of Uncertainty, Fuzziness and Knowledge-Based Systems, vol. 21, pp. 139-155, 2013.
[5] E. Szmidt, J. Kacprzyk, and P. Bujnowski, "How to measure the amount of knowledge conveyed by Atanassov's intuitionistic fuzzy sets," Information Sciences, vol. 257, pp. 276-285, 2013.

[6] C.-P. Wei, P. Wang, and Y.-Z. Zhang, "Entropy, similarity measure of interval-valued intuitionistic fuzzy sets and their applications," Information Sciences, vol. 181, no. 19, pp. 42734286, 2011.

[7] J. Li, G. Deng, H. Li, and W. Zeng, "The relationship between similarity measure and entropy of intuitionistic fuzzy sets," Information Sciences, vol. 188, pp. 314-321, 2012.

[8] K. Atanassov, Intuitionistic Fuzzy Sets: Theory and Applications, Physica, Wyrzburg, Germany, 1999.

[9] W.-L. Hung and M.-S. Yang, "On similarity measures between intuitionistic fuzzy sets," International Journal of Intelligent Systems, vol. 23, no. 3, pp. 364-383, 2008.

[10] Z. Yue, "Deriving decision maker's weights based on distance measure for interval-valued intuitionistic fuzzy group decision making," Expert Systems with Applications, vol. 38, no. 9, pp. 11665-11670, 2011.

[11] Z. Liang and P. Shi, "Similarity measures on intuitionistic fuzzy sets," Pattern Recognition Letters, vol. 24, no. 15, pp. 2687-2693, 2003.

[12] E. Szmidt and J. Kacprzyk, "Distances between intuitionistic fuzzy sets," Fuzzy Sets and Systems, vol. 114, no. 3, pp. 505-518, 2000.

[13] M. Xia and Z. S. Xu, "Some new similarity measures for intuitionistic fuzzy values and their application in group decision making," Journal of Systems Science and Systems Engineering, vol. 19, no. 4, pp. 430-452, 2010.

[14] Z.S. Xu and R. R. Yager, "Some geometric aggregation operators based on intuitionistic fuzzy sets," International Journal of General Systems, vol. 35, no. 4, pp. 417-433, 2006.

[15] Z. S. Xu, "Intuitionistic fuzzy aggregation operators," IEEE Transactions on Fuzzy Systems, vol. 15, no. 6, pp. 1179-1187, 2007.

[16] R. R. Yager, "OWA aggregation of intuitionistic fuzzy sets," International Journal of General Systems, vol. 38, no. 6, pp. 617641, 2009.

[17] J. Ye, "Multicriteria fuzzy decision-making method based on a novel accuracy function under interval-valued intuitionistic fuzzy environment," Expert Systems with Applications, vol. 36, no. 3, pp. 6899-6902, 2009.

[18] Z. S. Xu, "A method based on distance measure for intervalvalued intuitionistic fuzzy group decision making," Information Sciences, vol. 180, no. 1, pp. 181-190, 2010.

[19] C. Tan and X. Chen, "Intuitionistic fuzzy Choquet integral operator for multi-criteria decision making," Expert Systems with Applications, vol. 37, no. 1, pp. 149-157, 2010.

[20] Z. S. Xu and M. M. Xia, "Induced generalized intuitionistic fuzzy operators," Knowledge-Based Systems, vol. 24, no. 2, pp. 197-209, 2011.

[21] X. Yu and Z. S. Xu, "Prioritized intuitionistic fuzzy aggregation operators," Information Fusion, vol. 14, pp. 108-116, 2013. 
[22] R. M. Rodríguez, L. Martinez, and F. Herrera, "Hesitant fuzzy linguistic term sets for decision making," IEEE Transactions on Fuzzy Systems, vol. 20, no. 1, pp. 109-119, 2012.

[23] K. Atanassov and G. Gargov, "Interval valued intuitionistic fuzzy sets," Fuzzy Sets and Systems, vol. 31, no. 3, pp. 343-349, 1989.

[24] V. Torra, "Hesitant fuzzy sets," International Journal of Intelligent Systems, vol. 25, no. 6, pp. 529-539, 2010.

[25] V. Torra and Y. Narukawa, "On hesitant fuzzy sets and decision," in Proceedings of the 2009 18th IEEE International Conference on Fuzzy Systems, pp. 1378-1382, Jeju Island, Republic of Korea, August 2009.

[26] B. Zhu, Z. S. Xu, and M. Xia, "Dual hesitant fuzzy sets," Journal of Applied Mathematics, vol. 2012, Article ID 879629, 13 pages, 2012.

[27] F. Herrera and L. Martínez, "A 2-tuple fuzzy linguistic representation model for computing with words," IEEE Transactions on Fuzzy Systems, vol. 8, no. 6, pp. 746-752, 2000.

[28] Y. Dong, Y. Xu, H. Li, and B. Feng, “The OWA-based consensus operator under linguistic representation models using position indexes," European Journal of Operational Research, vol. 203, no. 2, pp. 455-463, 2010.

[29] F. Herrera and L. Martínez, "A model based on linguistic 2tuples for dealing with multigranular hierarchical linguistic contexts in multi-expert decision-making," IEEE Transactions on Systems, Man, and Cybernetics B: Cybernetics, vol. 31, no. 2, pp. 227-234, 2001.

[30] F. Herrera, L. Martínez, and P. J. Sánchez, "Managing nonhomogeneous information in group decision making," European Journal of Operational Research, vol. 166, no. 1, pp. 115-132, 2005.

[31] Z. S. Xu, "A method based on linguistic aggregation operators for group decision making with linguistic preference relations," Information Sciences, vol. 166, no. 1-4, pp. 19-30, 2004.

[32] Y.-J. Xu and Q.-L. Da, "Standard and mean deviation methods for linguistic group decision making and their applications," Expert Systems with Applications, vol. 37, no. 8, pp. 5905-5912, 2010.

[33] G. Klir and B. Yuan, Fuzzy Sets and Fuzzy Logic: Theory and Applications, Prentice Hall, Upper Saddle River, NJ, USA, 1995.

[34] E. P. Klement, R. Mesiar, and E. Pap, Triangular Norms, Kluwer Academic Publishers, Dordrecht, The Netherlands, 2000.

[35] Z. S. Xu, "Deviation measures of linguistic preference relations in group decision making," Omega, vol. 33, no. 3, pp. 249-254, 2005.

[36] Z. S. Xu, "Induced uncertain linguistic OWA operators applied to group decision making," Information Fusion, vol. 7, no. 2, pp. 231-238, 2006.

[37] O. Holder, “Über einen Mittelwertsatz," in Göttingen Nachrichten, pp. 38-47, 1889.

[38] J. L. W. V. Jensen, "Sur les fonctions convexes et les inégalités entre les valeurs moyennes," Acta Mathematica, vol. 30, no. 1, pp. 175-193, 1906.

[39] Z. S. Xu, "An overview of methods for determining OWA weights," International Journal of Intelligent Systems, vol. 20, no. 8, pp. 843-865, 2005.

[40] F. Herrera and E. Herrera-Viedma, "Linguistic decision analysis: steps for solving decision problems under linguistic information," Fuzzy Sets and Systems, vol. 115, no. 1, pp. 67-82, 2000. 


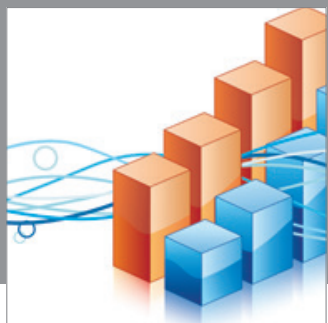

Advances in

Operations Research

mansans

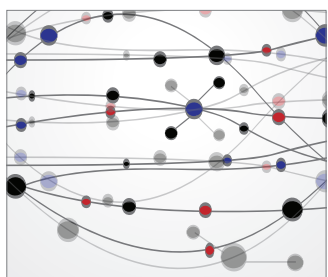

The Scientific World Journal
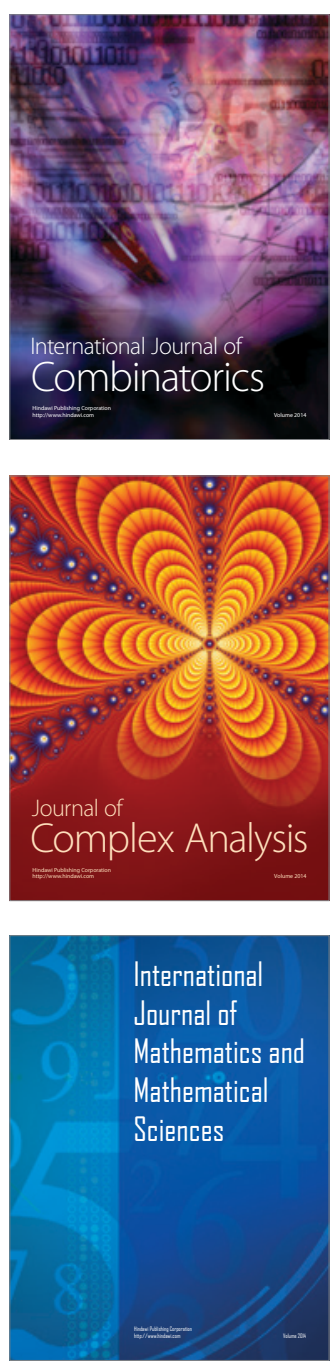
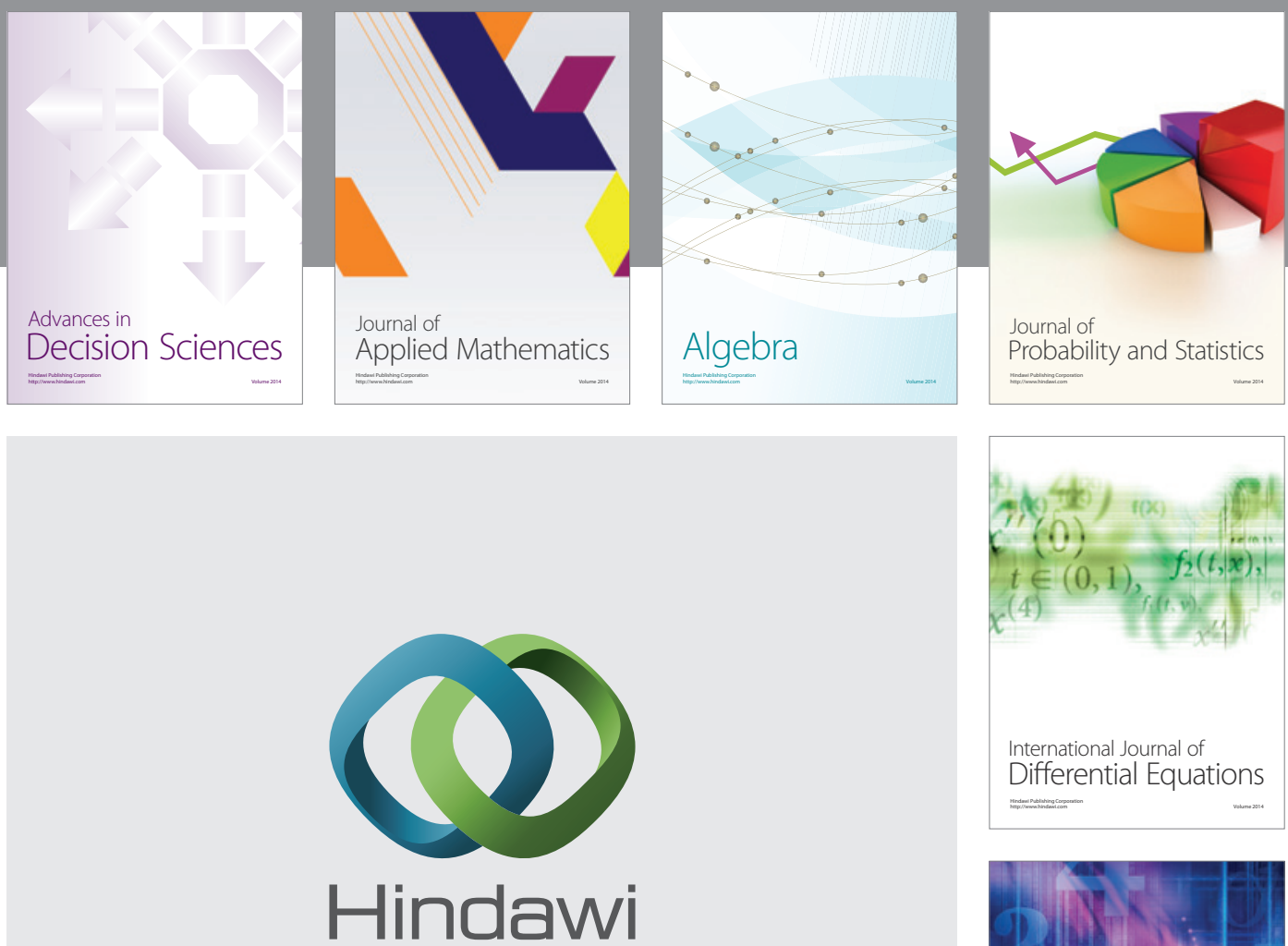

Submit your manuscripts at http://www.hindawi.com
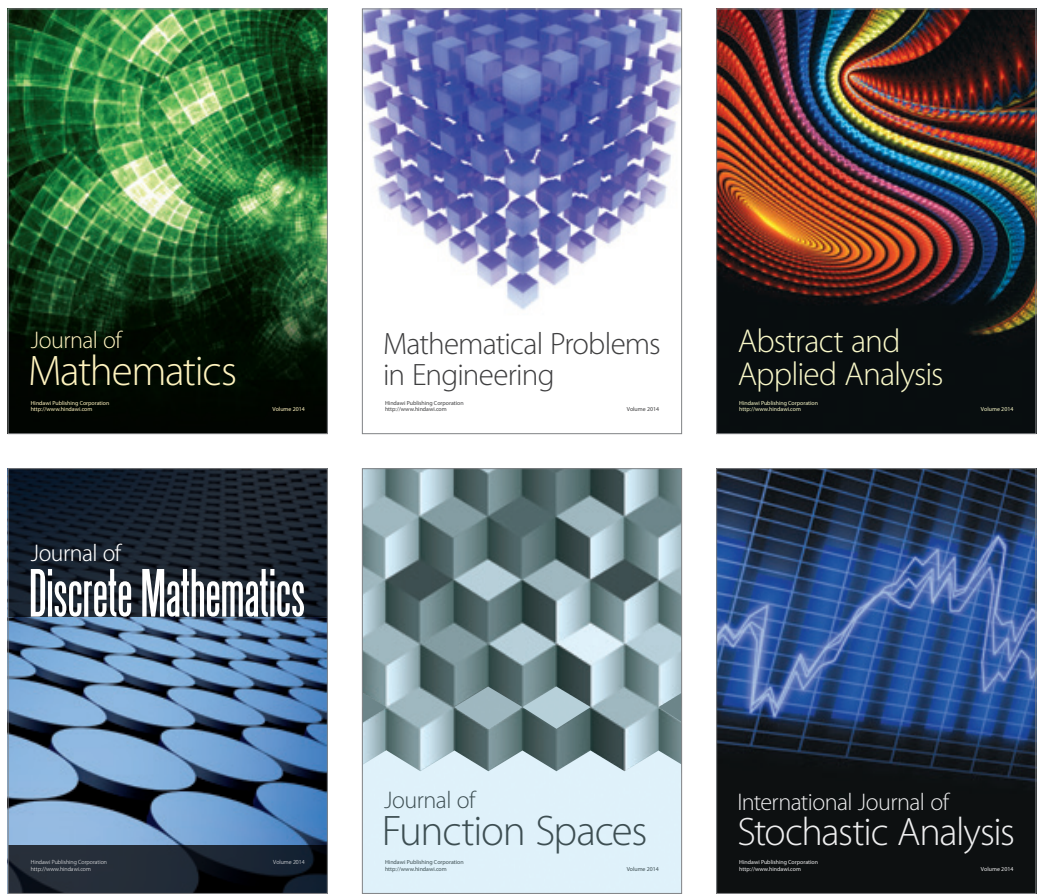

Journal of

Function Spaces

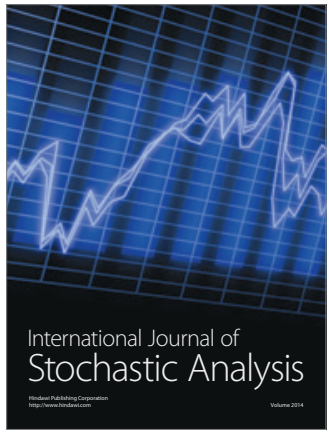

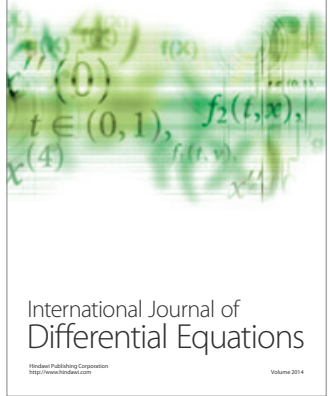
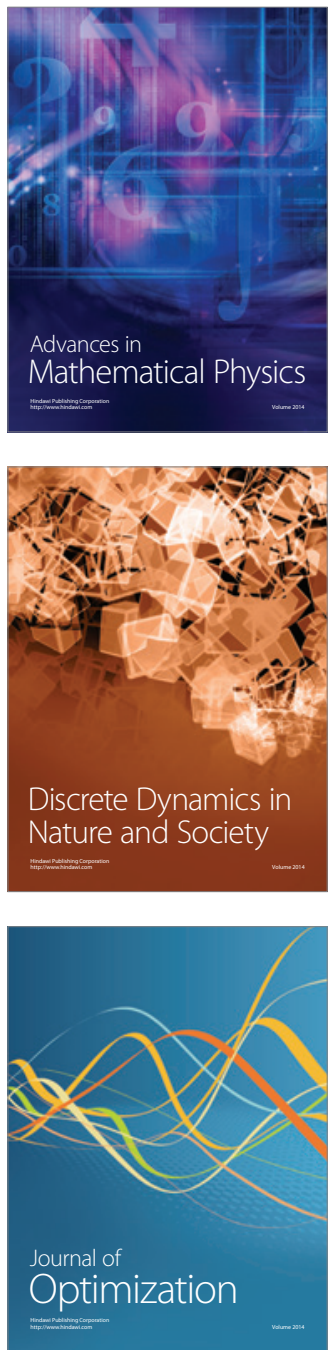